\title{
Impact of Eating Attitude and Impairment of Physical Quality of Life Between Tertiary Clinic and Primary Clinic Functional Dyspepsia Outpatients in Japan
}

\author{
Mayumi Shimpuku, ${ }^{1}$ Seiji Futagami, ${ }^{1 *}$ Natsuki Tajima, ${ }^{2}$ Hiroshi Yamawaki, ${ }^{1}$ Yuuta Maruki, ${ }^{1}$ Yasuhiro Kodaka, ${ }^{1}$ Hiroyuki Nagoya, ${ }^{1}$ \\ Katya Gudis, ${ }^{1}$ Tetsuro Kawagoe ${ }^{1}$ and Choitsu Sakamoto ${ }^{1}$
}

${ }^{1}$ Division of Gastroenterology, Department of Internal Medicine, Nippon Medical School, Tokyo, Japan; and ${ }^{2}$ Iri Clinic, Saitama, Japan

\begin{abstract}
Background/Aims
There is no available data on factors associated with healthcare-seeking behavior for functional dyspepsia (FD) symptoms at either tertiary or primary clinics in Japan. Therefore, we aimed to compare clinical symptoms and life styles such as sleep disorders and eating attitude in FD patients visiting general practitioners at primary clinics with those consulting gastroenterologists at tertiary clinics to clarify healthcare-seeking patterns in Japanese patients.
\end{abstract}

\section{Methods}

Fifty-one FD outpatients in a tertiary clinic (college hospital), 50 FD outpatients visiting primary clinics and 50 healthy volunteers were enrolled. Clinical symptoms, quality of life, sleep disorders, eating attitude and anxiety were estimated using the Gastrointestinal Symptom Rating Scale (GSRS), Social Functioning-8 (SF-8) test, Pittsburg Sleep Quality Index (PSQI) test and State-Trait Anxiety Inventory (STAI) for FD outpatients and healthy volunteers.

Results

FD outpatients exhibited higher mean scores of GSRS than healthy volunteers. The SF-8 physical component summary scores in the tertiary clinic group were significantly lower than those in the primary clinic group. GSRS scores were significantly $(P<$ $0.001, P=0.002)$ associated with global PSQI scores in FD outpatients as well as with STAl-trait scores $(P=0.006, P=$ $0.001)$ compared to healthy volunteers. The frequency of eating between meals in the primary clinic group was significantly $(P<0.05)$ higher than that in the tertiary clinic group.

Received: February 15, 2014 Revised: May 23, 2014 Accepted: May 25, 2014

(c) This is an Open Access article distributed under the terms of the Creative Commons Attribution Non-Commercial License (http://creativecommons. org/licenses/by-nc/3.0) which permits unrestricted non-commercial use, distribution, and reproduction in any medium, provided the original work is properly cited.

*Correspondence: Seiji Futagami, MD, PhD

Division of Gastroenterology, Department of Internal Medicine, Nippon Medical School, 1-1-5 Sendagi, Bunkyo-ku, Tokyo 113-8602, Japan

Tel: +81-3-3822-2131, Fax: +81-3-5685-1793, E-mail: seiji.futagami@gmail.com

Financial support: None.

Conflicts of interest: None.

Author contributions: Mayumi Shimpuku, data collection, data processing and data analysis; Seiji Futagami, study design, data collection and writing manuscript; Natsuki Tajima, data collection; Yuuta Maruki, Yasuhiro Kodaka, Hiroshi Yamawaki, Hiroyuki Nagoya and Tetsuro Kawagoe, data collection; Katya Gudis and Choitsu Sakamoto, writing manuscript.

ORCID: Mayumi Shimpuku, https://orcid.org/0000-0003-3727-5270; Seiji Futagami, http://orcid.org/0000-0003-0502-8646; Natsuki Tajima, http://orcid.org/0000-0001-9554-8105; Hiroshi Yamawaki, http://orcid.org/0000-0003-1427-2384; Yuuta Maruki, https://orcid.org/00000003-2155-0194; Yasuhiro Kodaka, http://orcid.org/0000-0002-5040-7955; Hiroyuki Nagoya, http://orcid.org/0000-0002-3240-2771; Katya Gudis, http://orcid.org/0000-0002-5832-7271; Tetsuro Kawagoe, http://orcid.org/0000-0003-3635-898X; Choitsu Sakamoto, http://orcid. org/0000-0002-1792-3165. 


\section{Conclusions}

It may be important for clarification of healthcare-seeking behavior to determine the difference in both impairment of physical quality of life and eating attitudes between tertiary clinic and primary clinic FD outpatients in Japan.

(J Neurogastroenterol Motil 2014;20:506-515)

\section{Key Words}

Anxiety; Eating attitude; Functional dyspepsia; Healthcare-seeking behavior; Sleep disorders; Quality of life

\section{Introduction}

Functional dyspepsia (FD) has been subdivided into 2 new disease categories based on the Rome III classification criteria: epigastric pain syndrome (EPS) and postprandial distress syndrome (PDS). ${ }^{1}$ The majority of $\mathrm{FD}$ patients complain of various symptoms related to the intake of meals and eating attitude; however, the pathophysiology of $\mathrm{FD}$ remains unclear in view of eating attitudes. ${ }^{2,3}$ In addition, sleep disturbance is a common medical problem. An epidemiological survey on sleep disordrs demonstrated that $17.3 \%$ to $22.3 \%$ of the general Japanese population suffer from sleep disorders. ${ }^{4-6}$ In addition, sleep disorders have been associated with several diseases, including pulmonary diseases, gastroesophageal reflux disease (GERD) and fibromyalgia. ${ }^{7}$ However, several studies have found a relationship between sleep disturbance and functional GI disorders. ${ }^{8-11}$ Gastrointestinal (GI) symptoms are some of the key factors affecting health-related quality of life (HRQOL). ${ }^{12,13}$ HRQOL is affected by wellness sensation which consists of good eating, good bowel movement and good sleep. These three factors also correlate with each other and, in turn, might be associated with healthcare-seeking behavior. Therefore, it is very important to determine whether these 3 factors affect healthcare-seeking behavior in FD patients.

Despite of the high incidence rate of dyspepsia in the community, the majority of dyspeptics do not consult with a medical practitioner for clinical symptoms. ${ }^{14,15}$ British surveys revealed that only $22-25 \%$ of people with dyspepsia have consulted a doctor for symptoms within the previous 6 or 12 months and only $42 \%$ ever sought medical advice. ${ }^{14}$ Previous studies have reported that factors such as an increasing age, female gender, duration of dyspepsia, severity of symptoms, frequent dyspepsia, lower socioeconomic status and experience of stressful life events are associated with consulting a doctor for dyspepsia. ${ }^{14-20}$ Understanding the proportions and profiles of the patients who consult with college gastroenterologists and general practitioners provides important information concerning the various factors affecting consultation choice, in turn to lead to clarify the etiology of FD patients. Therefore, in this study, we compared FD outpatients consulting gastroenterologists at a tertiary clinic with FD outpatients consulting general practitioners at a primary clinic using a variety of instruments including Gastrointestinal Symptom Rating Scale (GSRS), Pittsburg Sleep Quality Index (PSQI), Social Functioning-8 (SF-8) and State-Trait Anxiety Inventory (STAI) scores and eating attitude to determine the epidemiology and development of FD patients in Japan.

\section{Materials and Methods}

\section{Patients}

This study enrolled 51 consecutive patients consulting specialists in gastroenterology at the Nippon Medical School outpatient clinic (tertiary clinic) outpatient for typical symptoms of FD (EPS, $\mathrm{n}=25$; PDS, $\mathrm{n}=39$; EPS-PDS overlap, $\mathrm{n}=13$ ) and 50 consecutive patients (EPS, $\mathrm{n}=25$; PDS, $\mathrm{n}=30$; EPS-PDS overlap, $\mathrm{n}=5$ ) consulting nonspecialists at a primary clinic for the treatment of FD symptoms. FD outpatients can visit a tertiary clinic with recommendation documents from other hospitals. All patients were enrolled following upper GI endoscopy. Patients presented with various types of GI symptoms including nausea and upper abdominal discomfort, in addition to the four typical upper abdominal symptoms based on the Rome III classification: ${ }^{21}$ bothersome postprandial fullness, early satiation, epigastric pain and epigastric burning. Dyspeptic symptoms were defined as pain or discomfort in the upper abdomen for the past three months, with symptom onset at least six months prior to medical check-up. In addition, 50 healthy volunteers, with no clinical history of gastroduodenal disease including symptoms of FD, were recruited. Exclusion criteria included severe heart dis- 
eases, renal or pulmonary failure, liver cirrhosis, severe systemic illness, history of malignant diseases, and erosive GERD. Patients with previous gastroduodenal surgery, duodenal ulcer scars, diabetes mellitus, and recent use of non-steroidal anti-inflammatory drugs, proton pump inhibitors or anticoagulants at endoscopy were also excluded. Written informed consent was obtained from all subjects prior to upper GI endoscopy and abdominal ultrasonography for evaluation of dyspeptic symptoms. The study protocol was approved by the Ethics Review Committee of Nippon Medical School Hospital (approval number: 221029).

\section{Clinical Symptoms}

Abdominal symptoms were evaluated using a previously validated questionnaire. ${ }^{22-24}$ We estimated clinical symptoms with the GSRS. ${ }^{25}$ The GSRS is composed of 15 items (epigastric pain, epigastric burning, gastroesophageal reflux symptom, eructation, postprandial fullness, abdominal distention, early satiety, abdominal pain, the feeling of hunger, nausea, borborygmus, constipation, diarrhea, loose stools and hard stools) which generated 5 components including gastroesophageal reflux, abdominal pain, dyspepsia, diarrhea and constipation. Each item was rated according to severity on a scale of 1 (no discomfort at all) to 7 (very severe discomfort). We used the mean score of GSRS and the 15 GI symptoms of the GSRS for evaluating the dyspeptic symptoms. Gastroesophageal reflux symptom was defined as a burning feeling rising from the stomach or lower chest up toward the neck with a frequency of less than once per week (either 2-3 days a month, once a month, or less than once a month). The chief complaints of patients are centered around the frequency, duration and intensity of clinical symptoms associated with FD rather than GERD. The diagnoses of FD-non-erosive reflux disease (NERD) overlap syndrome, FD-irritable bowel syndrome (IBS) overlap syndrome, and FD-NERD-IBS overlap syndrome were indicated by the coexistence of symptoms of FD and NERD, of FD and IBS, and of FD, NERD and IBS, respectively.

Clinical symptoms after ingestion of a high-fat meal were scored as follows: 0 , no complaints; and 1, presence of any clinical symptom including gastroesophageal reflux, abdominal pain, dyspepsia, diarrhea and constipation.

\section{Pittsburgh Sleep Quality Index}

Sleep quality and sleep duration were evaluated by a Japanese version of the PSQI questionnaire. ${ }^{26}$ The PSQI questionnaire consists of 17 items that generate seven components, including subjective sleep quality, sleep latency, sleep duration, habitual sleep efficiency, sleep disturbance, use of sleep medication and daytime dysfunction. The score of each component ranges from 0 to 3 , reflecting severity of symptoms and the sum of the seven component scores provide a global PSQI score that ranges from 0 to 21 . Higher scores indicate poorer sleep. ${ }^{4,26}$ A cut-off score $>5.5$ has a sensitivity of $80.0-85.7 \%$ for various patient groups, and a specificity of $86.6 \%$ for control subjects in the Japanese version of the PSQI. ${ }^{4}$

\section{State-Trait Anxiety Inventory}

The State-Trait Anxiety Inventory (STAI) is a well-validated 40 -item self-reported questionnaire to estimate the severity of anxiety. ${ }^{27}$ The STAI is used to measure both state of anxiety (20 items) and trait of anxiety (20 items), wherein subjects choose one of four levels of anxiety for each item. State of anxiety reflects a "transitory emotional state or condition of the human organism that is characterized by subjective, consciously perceived feelings of tension and apprehension, and heightened autonomic nervous system activity." State of anxiety may fluctuate over time and can vary in intensity. In contrast, trait of anxiety denotes "relatively stable individual differences in anxiety proneness.”

\section{Health-related Quality of Life}

The SF-8 test was used to measure HRQOL according to the "Manual of the SF-8 Japanese Version." ${ }^{28}$ The SF-8 scores 8 domains, including general health, physical functioning, role-physical, bodily pain, vitality, social functioning, mental health and role-emotional that are then combined with a physical component summary (PCS) and a mental component summary (MCS) to arrive at a composite score. A score $<50$ thus indicates impaired quality of life (QOL), with lower scores considered to indicate greater damage to QOL. Eating attitude was evaluated according to the frequency of eating between meals and the proportion of dinners taken at midnight.

\section{Statistical Methods}

For statistical evaluation of group data, Student's $t$ test for paired data were followed by Scheffe's F test for continuous variables. For categorical data, Chi-squared test was used. To characterize the healthcare-seeking behavior, i.e., the tendency for FD patients to prefer to choose a tertiary clinic rather than primary clinics, logistic regression analysis was performed using a variety of factors we analyzed in the present study. The data analyses were performed using a standard software package (SPSS version 13.0, Chicago, IL, USA). A $P$-value of $<0.05$ was statistically significant. 


\section{Results}

\section{Characteristics of Tertiary and Primary Clinic Functional Dyspepsia Outpatients}

The characteristics of tertiary and primary clinic FD patients including age, sex, body mass index and smoking rate did not differ significantly from those of healthy volunteers (Table 1). Mean scores of GSRS in tertiary clinic and primary clinic FD

Table 1. Characteristics of Tertiary Clinic and Primary Clinic Functional Dyspepsia Outpatients

\begin{tabular}{lclcc}
\hline & $\begin{array}{c}\text { Age } \\
\text { (range) }\end{array}$ & $\begin{array}{c}\text { Sex } \\
(\mathrm{M} / \mathrm{F})\end{array}$ & $\begin{array}{c}\text { BMI } \\
(\text { mean } \pm \mathrm{SE})\end{array}$ & $\begin{array}{c}\text { Smoking } \\
\text { rate }(\%)\end{array}$ \\
\hline Tertiary clinic FD & $24-89$ & $21 / 30$ & $22.08 \pm 0.59$ & 18.8 \\
Primary clinic FD & $23-83$ & $38 / 2$ & $22.46 \pm 0.46$ & 20.0 \\
Healthy volunteers & $26-83$ & $14 / 36$ & $22.00 \pm 0.60$ & 30.0
\end{tabular}

$\mathrm{BMI}$, body mass index; FD, functional dyspepsia.

A

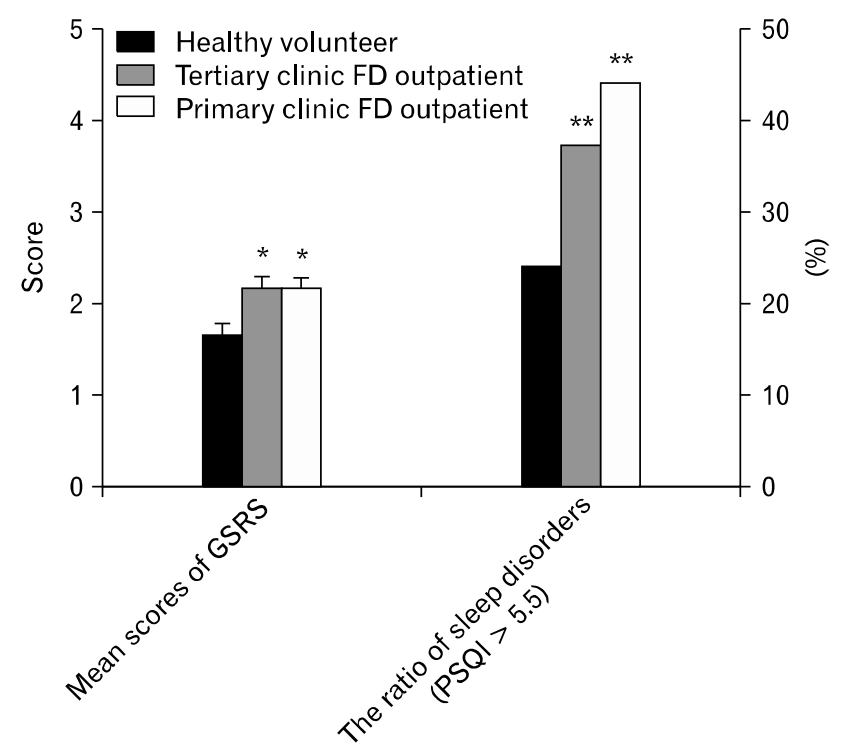

outpatients (2.17 \pm 0.12 and $2.18 \pm 0.09$, respectively) were significantly higher $(P<0.001$ and $P<0.001$, respectively) than those in healthy volunteers (Fig. 1A). In subgroup analysis of GSRS scores, scores for gastroesophageal reflux, abdominal pain, dyspepsia and constipation were significantly higher in both tertiary clinic and primary clinic FD outpatients than healthy volunteers (Fig. 1B). However, diarrhea scores did not differ significantly across groups (Fig. 1B). In addition, sleep disorder, defined as global PSQI scores $>5.5$ was found in 19 out of 51 (37.3\%) tertiary clinic FD outpatients and in 22 of the $50(44 \%)$ primary clinic FD outpatients (Fig. 1A). In contrast, global PSQI scores $>5.5$ were found in 12 of 50 (24\%) healthy volunteers. The prevalence of sleep disorders in tertiary clinic FD outpatients was not significantly $(P=0.334)$ higher than that in primary clinic FD outpatients. In contrast, the prevalence of sleep disorders in either tertiary clinic or primary clinic FD outpatients was significantly $(P<0.05)$ higher than that in healthy volunteers (Fig. 1A).

SF-8 (PCS) scores of FD patients in tertiary clinic and primary clinic outpatients were significantly lower than in healthy
B

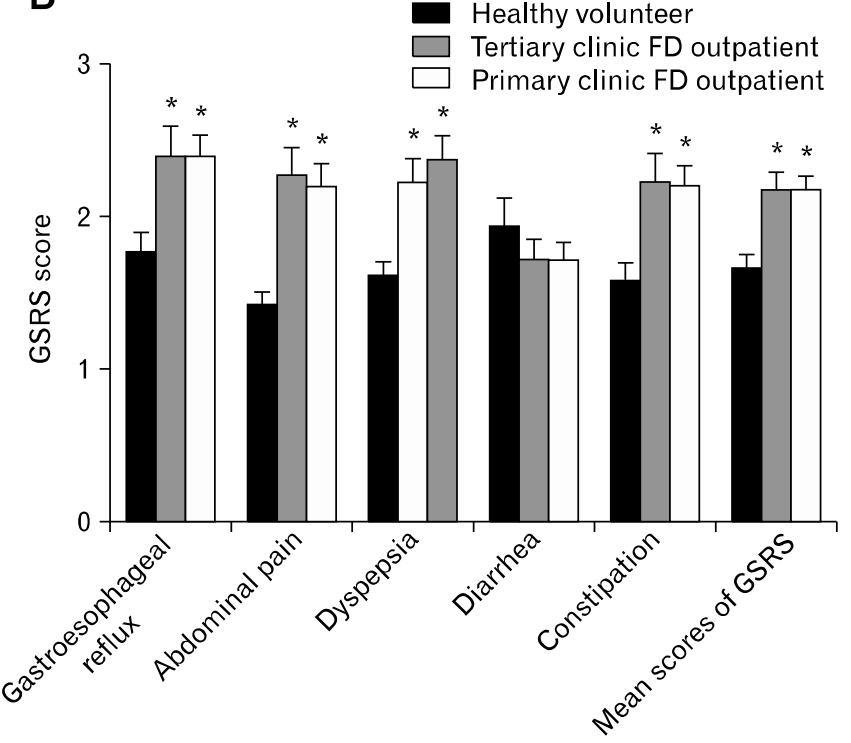

Figure 1. Clinical symptoms and sleep disorders among tertiary clinic functional dyspepsia (FD) outpatients, primary clinic FD outpatients and healthy volunteers. (A) Mean scores of Gastrointestinal Symptoms Rating Scale (GSRS) and the ratio of sleep disorders (Pittsburgh Sleep Quality Index [PSQI] score > 5.5) among tertiary clinic FD outpatients, primary clinic FD outpatients and healthy volunteers. Mean scores of GSRS in tertiary clinic and primary clinic FD outpatients were significantly higher compared to that in healthy volunteers. ${ }^{*} P<0.001$ vs. healthy volunteers. The ratio of sleep disorders (PSQI score $>5.5$ ) in tertiary clinic and primary clinic FD outpatients were significantly higher compared to that in healthy volunteers, ${ }^{* *} P<0.05$ vs. healthy volunteers. (B) Each GSRS score among tertiary clinic FD outpatients, primary clinic outpatients and healthy volunteers. Scores of gastroesophageal reflux, abdominal pain, dyspepsia and constipation in tertiary clinic and primary clinic outpatients were significantly higher compared to healthy volunteers. ${ }^{*} P<0.001$ vs. healthy volunteers. 
volunteers (Fig. 2). The SF-8 (PCS) score of FD patients was significantly lower in tertiary clinic than in primary clinic FD outpatients (Fig. 2). However, the SF-8 (MCS) scores of FD patients did not differ significantly among the three groups (Fig. 2). STAI (state and trait) scores in tertiary clinic FD outpatients (53.51 \pm 2.94 and $49.86 \pm 3.61$, respectively) and primary clinic FD outpatients $(52.76 \pm 4.34$ and $44.02 \pm 4.20$, respectively) were significantly higher than in healthy volunteers (Fig. 2). In contrast, there was no significant difference in either the STAI-state or the STAI-trait scores between tertiary clinic and primary clinic FD outpatients (Fig. 2). The percentages of duration of the disease in tertiary clinic were similar to those in primary clinic FD outpatients.

\section{Comparison of Overlap Syndrome in Tertiary Clinic and Primary Clinic Functional Dyspepsia Outpatients}

The ratios of $\mathrm{FD}$ only, $\mathrm{FD} /$ non-erosive reflux disease (NERD) overlap, FD/irritable bowel syndrome (IBS) overlap and $\mathrm{FD} / \mathrm{NERD} / \mathrm{IBS}$ overlap were $53 \%, 16 \%, 17 \%$ and $14 \%$, respectively, in tertiary clinic outpatients (Fig. 3). In contrast, in primary clinic FD outpatients, the ratios of FD only, FD/NERD overlap, FD/IBS overlap and FD/NERD/IBS overlap were

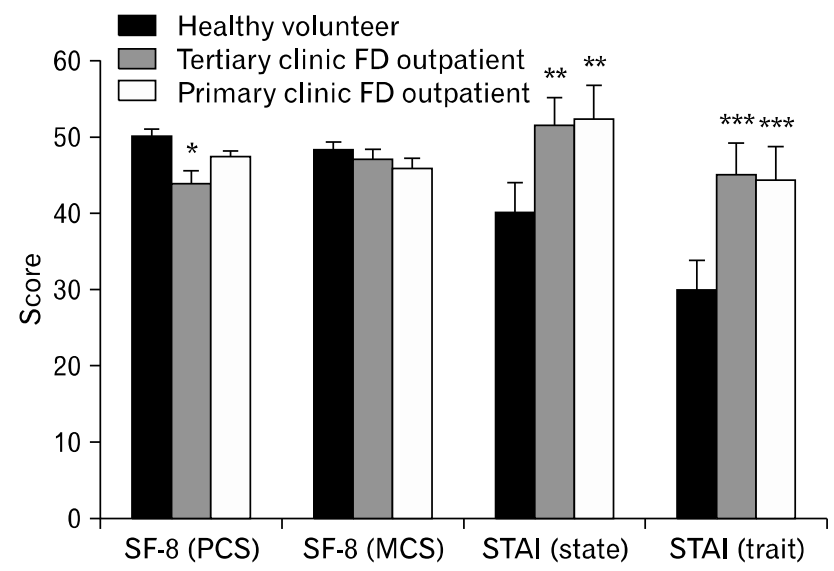

Figure 2. Quality of life and anxiety in tertiary clinic and primary clinic functional dyspepsia (FD) outpatients Social Functioning-8 physical component summary (SF-8 [PCS]) score in FD outpatients in tertiary clinic was significantly lower compared to those in primary clinic FD outpatients and healthy volunteers. ${ }^{*} P<0.001$ vs. healthy volunteers and primary clinic FD outpatients. State-Trait Anxiety Inventory (STAI) scores in tertiary clinic and primary clinic FD outpatients were significantly higher compared to those in healthy volunteers, ${ }^{*} P<$ 0.001 vs. STAI-state scores of healthy volunteers, ${ }^{* \star *} P<0.001$ vs. STAI-trait scores of healthy volunteers. MCS, mental component summary.
$46 \%, 22 \%, 18 \%$ and $14 \%$, respectively (Fig. 3). The overall ratio of overlap syndrome in FD outpatients at the tertiary clinic was similar $(P=0.322)$ to that in primary clinic FD outpatients. The ratio of FD-NERD overlap syndrome in tertiary clinic outpatients was almost similar to that in primary clinic FD outpatients. In addition, the ratio of FD-IBS overlap syndrome in tertiary clinic outpatients was also similar to that in primary clinic outpatients (Fig. 3). The symptoms score of FD-IBS overlap syndrome $(\mathrm{n}=16,2.97 \pm 0.223)$ in tertiary clinic FD outpatients was not significantly $(P=0.306$ ) higher compared to that in primary clinic FD outpatients $(n=16,2.73 \pm 0.147)$.

\section{Comparison of Pittsburg Sleep Quality Index Score in Tertiary Clinic and Primary Clinic Functional Dyspepsia Outpatients with Sleep disorders}

The mean \pm SD PSQI scores of patients with sleep disorder $(\mathrm{PSQI}$ score $>5.5)$ were $8.53 \pm 0.51,8.68 \pm 0.46$ and $6.67 \pm$ 0.22 among tertiary clinic FD outpatients, primary clinic FD outpatients and healthy volunteers, respectively. The mean value of PSQI score in tertiary clinic FD outpatients with sleep disorders (PSQI score $>5.5)$ was not significantly $(P=0.822)$ different compared to that in primary clinic FD outpatients with sleep disorders. The mean values of PSQI score in tertiary clinic and primary clinic FD outpatients with sleep disorders were significantly ( $P=0.009, P=0.004$, respectively) higher compared to that in healthy volunteers with sleep disorders. In sub-analysis of PSQI scores, sleep latency, sleep disturbance and use of sleep medication in both tertiary clinic and primary clinic FD outpatients were significantly higher than in healthy volunteers $(P=$ 0.001, $P=0.031 ; P<0.001, P=0.007 ; P=0.001, P<$

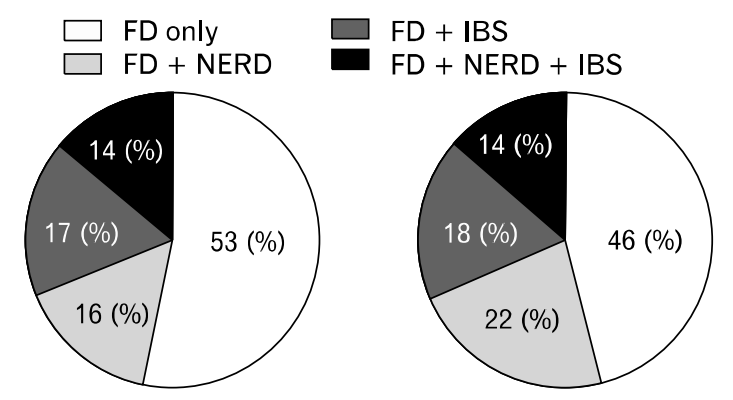

Tertiary clinic FD outpatient Primary clinic FD outpatient

Figure 3. The proportion of overlap syndrome in functional dyspepsia (FD) outpatients. The proportion of overlap syndrome in tertiary clinic and primary clinic FD outpatients. NERD, non-erosive reflux disease; IBS, irritable bowel syndrome. 
A

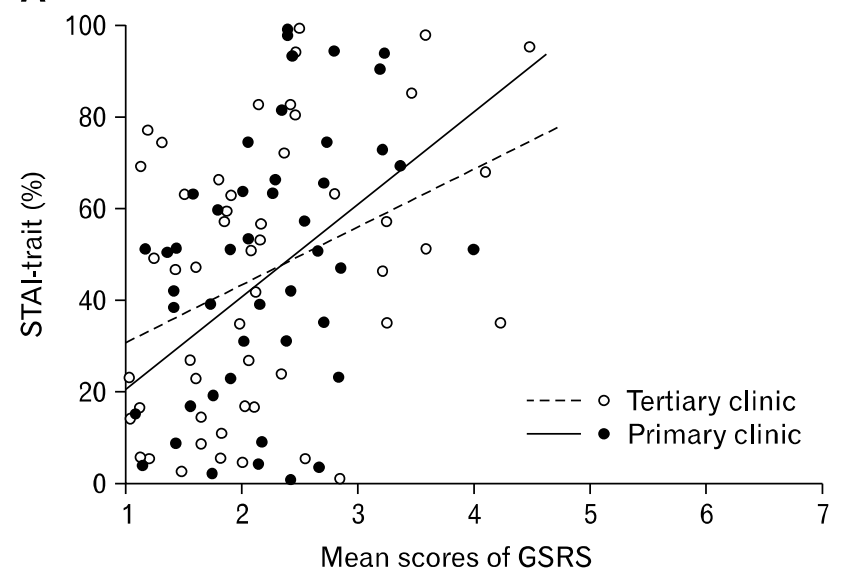

B

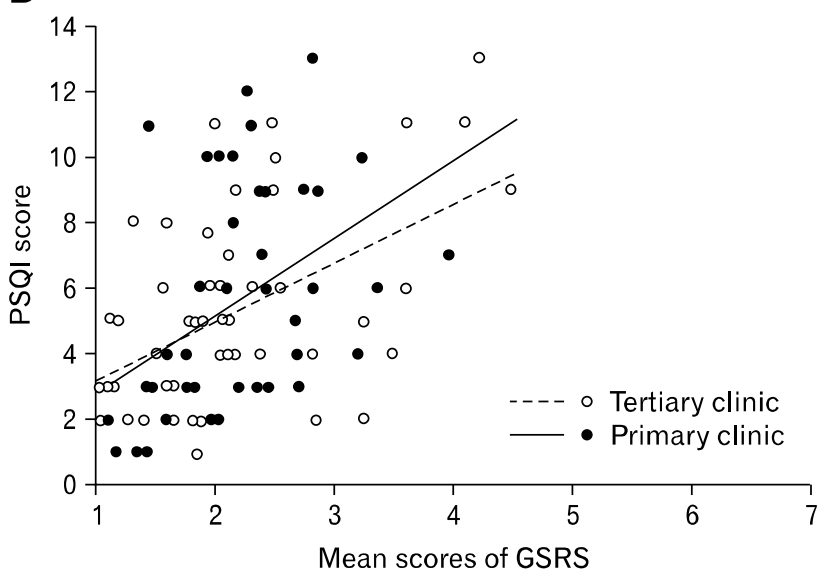

Figure 4. Correlations among mean scores of Gastrointestinal Symptoms Rating Scale (GSRS), global Pittsburgh Sleep Quality Index (PSQI) and State-Trait Anxiety Inventory (STAI)-trait scores in tertiary clinic and primary clinic functional dyspepsia (FD) outpatients. (A) The relationship between mean scores of GSRS and STAI-trait (\%) scores in tertiary clinic and primary clinic FD outpatients. Mean scores of GSRS were significantly $(P=0.006, P=0.001)$ associated with STAI-trait $(\%)$ scores in tertiary clinic and primary clinic FD patients. (B) Global PSQI scores were significantly $(P<0.001, P=0.002)$ associated with mean scores of GSRS in tertiary clinic and primary clinic outpatients.

0.001 , respectively). In addition, habitual sleep disturbance scores $(P=0.013)$ were significantly higher in primary clinic FD outpatients than in healthy volunteers (Supplementary Table).

\section{Correlations Among Mean Score of} Gastrointestinal Symptoms Rating Scale, Global Pittsburgh Sleep Quality Index and State-Trait Anxiety Inventory-Trait Scores in Tertiary Clinic and Primary Clinic Functional Dyspepsia Outpatients

Since psychological factors are known to play a critical role in FD symptoms, we examined the association between the mean score of GSRS and STAI-trait scores. STAI-trait scores in tertiary clinic and primary clinic FD outpatients significantly $(P=$ $0.006, \mathrm{R}^{2}=0.143 ; P=0.001, \mathrm{R}^{2}=0.192$, respectively) associated with mean score of GSRS (Fig. 4A). Global PSQI scores were significantly $\left(P<0.001, \mathrm{R}^{2}=0.254 ; P=0.002, \mathrm{R}^{2}=\right.$ 0.188 , respectively) association between the mean score of GSRS in tertiary clinic and primary clinic FD outpatients compared to healthy volunteers (Fig. 4B).

\section{Multiple Logistic Regression Analysis of Healthcare-seeking Behavior for Functional Dyspepsia Symptoms}

Since there is little available data regarding the healthcareseeking behavior for FD symptoms in Japan, we analyzed which of the following parameters were independently associated with
Table 2. Multiple Logistic Regression Analysis of Healthcareseeking Behavior for Functional Dyspepsia Symptoms

\begin{tabular}{lcc}
\hline \multicolumn{1}{c}{ Factors } & OR $(95 \% \mathrm{CI})$ & $P$-value \\
\hline Age $(\mathrm{yr})$ & $1.008(0.984-1.032)$ & 0.517 \\
Sex & $0.603(0.262-1.389)$ & 0.235 \\
EPS/PDS & $0.751(0.427-1.322)$ & 0.321 \\
BMI & $1.033(0.784-1.362)$ & 0.816 \\
Mean score of GSRS & $0.730(0.212-2.508)$ & 0.617 \\
Presence of IBS & $1.029(0.445-2.381)$ & 0.946 \\
Presence of GERD & $1.350(0.586-3.110)$ & 0.481 \\
PSQI score (> 5.5) & $1.482(0.662-3.320)$ & 0.914 \\
PCS & $1.062(1.005-1.123)$ & 0.032 \\
MCS & $0.966(0.911-1.025)$ & 0.253 \\
STAI-state & $0.992(0.971-1.014)$ & 0.492 \\
STAI-trait & $1.001(0.981-1.020)$ & 0.945 \\
\hline
\end{tabular}

EPS, epigastric pain syndrome; PDS, postprandial distress syndrome; BMI, body mass index; GSRS, Gastrointestinal Symptoms Rating Scale; IBS, irritable bowel syndrome; GERD, gastroesophageal reflux disease; PSQI, Pittsburgh Sleep Quality Index; PCS, physical component summary; MCS, mental component summary; STAI, State-Trait Anxiety Inventory.

the tendency to prefer to a tertiary over a primary clinic: age, sex, subtypes of FD, body mass index, mean score of GSRS, presence of IBS symptom, presence of GERD symptom, PSQI score ( $>$ 5.5), PCS, MCS, SF-8, STAI-state and STAI-trait. Multiple logistic regression analysis revealed that PCS were significantly $(P=0.032)$ associated with healthcare-seeking behavior of FD patients in Japan (Table 2). 


\section{Differences in Eating Attitude Between Tertiary Clinic and Primary Clinic Functional Dyspepsia Outpatients}

There was no significant difference in the proportion of patients taking dinner at midnight among healthy volunteers and FD outpatients (Fig. 5A). Interestingly, the frequency of eating between meals in primary clinic FD outpatients was significantly $(P<0.05)$ higher than that in tertiary clinic FD outpatients (Fig. 5B). In addition, the frequency of eating between meals in both tertiary clinic and primary clinic FD outpatients was significantly higher than that in healthy volunteers (Fig. 5B). Furthermore, intakes of fatty diet significantly aggravated clinical symptoms in both tertiary clinic and primary clinic FD outpatients compared to healthy volunteers (Fig. 5C). There was a significant $(P<0.05)$ difference in clinical symptoms induced by fat intakes between tertiary clinic and primary clinic FD outpatients (Fig. 5C). Since eating attitude has been reported to be

A
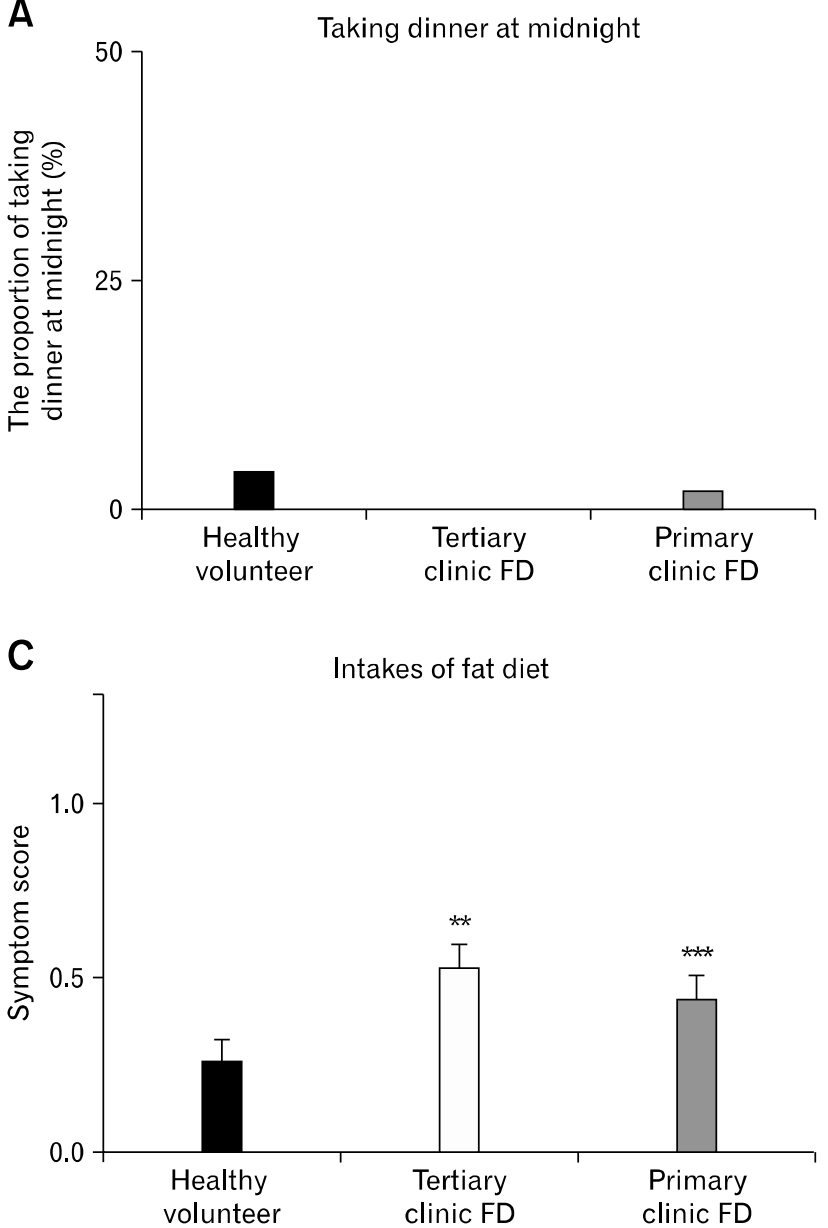

associated with sleep disorders, we also investigated the relationship between global PSQI scores and eating attitude in FD patients. The frequency of eating between meals in tertiary clinic FD outpatients was not significantly associated with the global PSQI score. In contrast, there was a relative trend $(P=0.072)$ to the relationship between intake of fat and the global PSQI score in tertiary clinic FD outpatients. In primary clinic FD outpatients, there was no significant relationship between either the frequency of eating between meals or intakes of fatty diet and global PSQI scores.

\section{Discussion}

The main findings of the present study are: (1) Mean scores of GSRS and global PSQI scores in tertiary clinic and primary clinic FD patients were significantly higher compared to those in healthy volunteers. In contrast, there was no significant difference in mean scores of GSRS or global PSQI scores between ter-

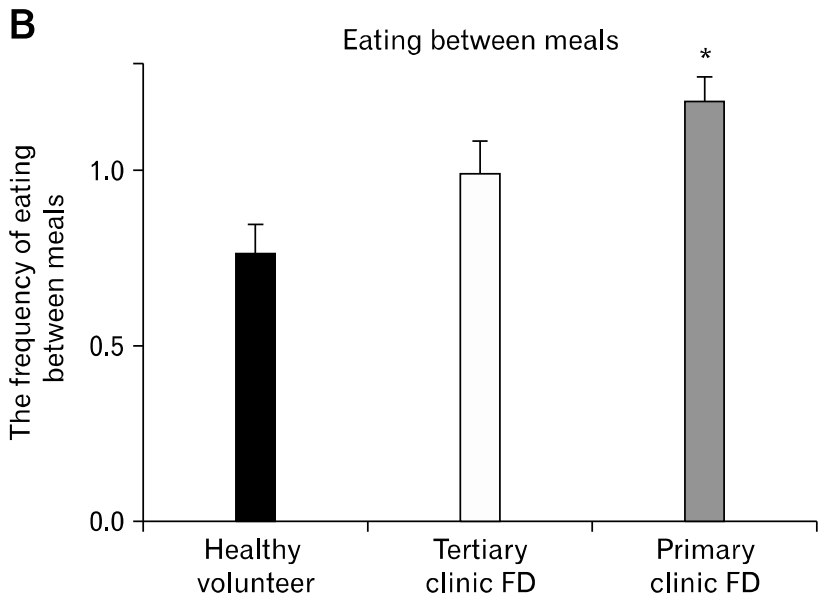

Figure 5. Differences in eating attitude in tertiary clinic and primary clinic functional dyspepsia (FD) outpatients. (A) There is no significant difference in the proportion of taking dinner at midnight among healthy volunteers and FD outpatients. (B) There is a significant difference in the frequency of eating between meals among tertiary clinic and primary clinic FD outpatients and healthy volunteers. The frequency of eating between meals in primary clinic FD outpatients was significantly higher compared to that in tertiary clinic FD outpatients. ${ }^{*} P<0.05$ vs. healthy volunteers and tertiary clinic FD outpatients. (C) In addition, intakes of fat diet significantly aggravated clinical symptoms in tertiary clinic and primary clinic FD outpatients compared to those of healthy volunteers. There was a significant difference in clinical appearance induced by fat intakes between tertiary clinic and primary clinic FD outpatients. ${ }^{* *} P$ $<0.05$ vs. healthy volunteers and primary clinic FD outpatients, ${ }^{* * *} P$ $<0.05$ vs. healthy volunteers. 
tiary clinic and primary clinic FD outpatients. (2) Impairment of physical quality of life significantly associated with healthcare-seeking behavior in FD patients in Japan. (3) The frequency of eating between meals in primary clinic FD outpatients was significantly higher compared to that in tertiary clinic FD outpatients.

Up to half of people experiencing symptoms associated with functional gastrointestinal disorders (FGIDs) never consult a physician despite the extended duration of their symptom. ${ }^{29,30}$ Healthcare-seeking behavior is complex and several studies have reported healthcare-seeking behavior for dyspepsia. ${ }^{14-20}$ Previous studies have reported that age, sex, increased symptom severity, increased symptom frequency, presence of IBS and increased anxiety were associated with consultation for dyspepsia. ${ }^{14-18}$ In contrast, in several reports, these factors including increased symptom severity and increased symptom frequency were not linked to consultation for dyspepsia. ${ }^{18-20,31}$ In Japan, there is no available data on factors associated with healthcare-seeking behavior for FD symptoms at either tertiary or primary clinics. We have also shown in this study that there is no significant difference between the spectrum of clinical symptoms between tertiary clinic and primary clinic FD outpatients in Japan. Previous studies have reported that patients with psychological comorbidities are more likely to seek medical attention for symptoms associated with FGID. ${ }^{32,33}$ In contrast, $\mathrm{Hu}$ et $\mathrm{al}^{18}$ have demonstrated that increased depression is not linked to consultation for dyspepsia. We could also find no significant difference between tertiary clinic and primary clinic FD outpatients for STAI-trait scores, sleep disorders or regularity of meal intake. In contrast, we found a significant difference in SF-8 PCS scores between tertiary clinic and primary clinic FD outpatients. In our data, SF-8 (PCS) is an important factor that affects healthcare-seeking behavior for FD symptoms in multiple logistic regression analysis in Table 2. These findings suggest that FD outpatients whose GI symptoms limit or compromise their physical activity are more likely to consult with a gastroenterologist rather than a general practitioner. Previous studies have reported that QOL affected by eating attitude, sleeping disorders and clinical symptoms was associated with severity of diseases in FD patients. ${ }^{13,34}$ Kaji et al ${ }^{35}$ have also reported that QOL was particularly disturbed in the PCS for FD-NERD overlap syndrome and in the MCS for FD-IBS overlap syndrome. These studies and our own results suggest that the PCS, one component of SF-8 score influenced by clinical symptoms, sleep disorders and eating attitude might affect a patient's decision to seek healthcare at a tertiary clinic. Further studies will be needed to investigate why consultation behavior for tertiary clinic FD outpatients was associated with the impairment of physical quality of life.

Hongo $^{13}$ found that the development of FGID symptoms correlated with improper diet, impaired bowel movement and poor sleep. Miwa ${ }^{36}$ addressed a negative relationship between regular meal intake and the development of FD and IBS symptoms. In a similar study, Shinozaki et $\mathrm{al}^{37}$ showed that Japanese outpatients with IBS had a higher frequency of irregular meal intake than did patients without IBS. However, in our data, there was no significant difference in the prevalence of subjects taking dinner at midnight among healthy volunteers, tertiary clinic and primary clinic FD outpatients. Interestingly, the frequency of eating between meals in primary clinic FD outpatients was significantly higher compared to that in tertiary clinic FD outpatients. Previous study has reported that the overnight fast time was significantly greater in FD patients compared to that in controls because FD patients tend to avoid food at night out of fear of developing abdominal discomfort. ${ }^{38}$ Their study reported that the daytime fast was similar in both groups. Pilichiewicz et $\mathrm{al}^{38}$ speculated that the frequency of eating between meals might be partly affected by the severity of FD symptoms. In contrast, irregular eating patterns might reflect irregular lifestyles, including irregular feeding times, sleeping and waking times, as well as working hours. In addition, poor eating habits such as skipping breakfast or lunch, and snacking while performing other tasks, could also be involved in the symptomology of FD. Taking into consideration the lack of any significant difference in the severity of symptoms between tertiary clinic and primary clinic FD outpatients, irregular life styles might explain the discrepancy in the frequency of eating between meals in the 2 groups.

In addition, our study shows that intakes of fat diet aggravates clinical symptoms in tertiary clinic and primary clinic FD outpatients compared to healthy volunteers similar with a previous report. Pilichiewicz et $\mathrm{al}^{39}$ report that high fat intake induces nausea and abdominal pain in FD patients compared to healthy volunteers. In our study, there was a significant difference between tertiary clinic and primary clinic FD outpatients in their symptomatic response to fat intake. Cuperus et al have also reported that eating patterns for the consumption of fat differed among FD populations. Whereas FD patients have a tendency to consume less fat during the day, there is a significant increase in their intake of fat at nighttime. ${ }^{32}$ Therefore, further studies will be needed to determine whether tertiary clinic and primary clinic FD outpatients differ significantly in their intake of fat during evening meals. 
It is possible that both sleep disorders and functional GI disorders might result from an underlying psychological problem, such as depression or anxiety. Previous study has demonstrated that state-anxiety is significantly and negatively correlated with discomfort and pain threshold. ${ }^{41}$ An epidemiological survey on insomnia demonstrated that from $17.3 \%$ to $22.3 \%$ of the general Japanese population suffer from sleep disorders. ${ }^{4-6}$ In our study, the prevalence of sleep disorders in $\mathrm{FD}$ patients visiting tertiary clinic $(37.3 \%)$ or primary clinic $(44 \%)$ was significantly higher than in healthy volunteers (24\%). Miwa ${ }^{36}$ have also reported that the prevalence of subjects who reported adequate sleep was significantly lower in FD/IBS patients than in control subjects. Lacy et $\mathrm{al}^{42}$ have reported that PSQI scores were higher in the patients with FD accompanied with moderate and severe symptoms. Fass et $\mathrm{al}^{8}$ have also reported a significant correlation between sleep disorders and perceived sensitivity of clinical symptoms for FD patients but not for IBS patients. Similarly, our data shows that total GSRS scores in both tertiary clinic and primary clinic FD outpatients significantly linked to the global PSQI scores. However, it remains to be clarified whether GI symptoms lead to sleep disorders or whether it is the sleep disorders that induce GI symptoms.

In addition, a recent large-scale epidemiologic study conducted in Japan found that participants who regarded their own diet as unhealthy had a higher prevalence of GI symptoms. ${ }^{13}$ These results suggest that eating attitudes and diet may be associated with the pathophysiology of FD and IBS. Although to date there have been but few reports on the clinical symptoms and diet of FGIDs patients, reports that specific foods and types of food are related to symptoms have begun to appear. ${ }^{43-45}$ In our study, there was significant difference in physical quality of life and eating attitudes in two different FD populations such as tertiary clinic and primary clinic FD outpatients. Further studies will be needed to investigate the difference in both impairment of physical quality of life and eating attitudes between tertiary clinic and primary clinic FD outpatients.

\section{Supplementary Material}

Note: To access the supplementary table mentioned in this article, visit the online version of Journal of Neurogastroenterology and Motility at http://www.jnmjournal.org/, and at doi: http://dx. doi.org/10.5056/jnm14015.

\section{References}

1. Tack J, Talley NJ, Camilleri M, et al. Functional gastroduodenal disorders. Gastroenterology 2006;130:1466-1479.

2. Talley NJ, Zinsmeister AR, Schleck CD, Melton LJ 3rd. Dyspepsia and dyspepsia subgroups: a population-based study. Gastroenterology 1992;102(4 Pt 1):1259-1268.

3. Castillo EJ, Camilleri M, Locke GR, et al. A community-based, controlled study of the epidemiology and pathophysiology of dyspepsia. Clin Gastroenterol Hepatol 2004;2:985-996.

4. Doi Y, Minowa M, Okawa M, Uchiyama M. Prevalence of sleep disturbance and hypnotic medication use in relation to sociodemographic factors in the general Japanese adult population. J Epidemiol 2000;10:79-86.

5. Kim K, Uchiyama M, Liu X, et al. Somatic and psychological complaints and their correlates with insomnia in the Japanese general population. Psychosom Med 2001;63:441-446.

6. Kim K, Uchiyama M, Okawa M, Liu X, Ogihara R. An epidemiological study of insomnia among the Japanese general population. Sleep 2000;23:41-47.

7. Parish JM. Sleep-related problems in common medical conditions. Chest 2009;135:563-572.

8. Fass R, Fullerton S, Tung S, Mayer EA. Sleep disturbances in clinic patients with functional bowel disorders. Am J Gastroenterol 2000; 95:1195-2000.

9. Jarrett M, Heitkemper M, Cain KC, Burr RL, Hertig V. Sleep disturbance influences gastrointestinal symptoms in women with irritable bowel syndrome. Dig Dis Sci 2000;45:952-959.

10. Goldsmith G, Levin JS. Effect of sleep quality on symptoms of irritable bowel syndrome. Dig Dis Sci 1993;38:1809-1814.

11. David D, Mertz H, Fefer L, et al. Sleep and duodenal motor activity in patients with severe non-ulcer dyspepsia. Gut 1994;35:916-925.

12. Chang L. Review article: epidemiology and quality of life in functional gastrointestinal disorders. Aliment Pharmacol Ther 2004;20(suppl 7):31-39.

13. Hongo M. Epidemiology of FGID symptoms in Japanese general population with reference to life style. J Gastroenterol Hepatol 2011; 26(suppl 3):19-22.

14. Jones RH, Lydeard SE, Hobbs FD, et al. Dyspepsia in England and Scotland. Gut 1990;31:401-405.

15. Jones R, Lydeard S. Prevalence of symptoms of dyspepsia in the community. BMJ 1989;298:30-32.

16. Ford AC, Forman D, Bailey AG, Cook MB, Axon AT, Moayyedi P. Who consults with dyspepsia? Results from a longitudinal 10-yr follow-up study. Am J Gastroenterol 2007;102:957-965.

17. Talley NJ, Boyce $P$, Jones M. Dyspepsia and health care seeking in a community: how important are psychological factors? Dig Dis Sci 1998;43:1016-1022.

18. Hu WH, Wong WM, Lam CL, et al. Anxiety but not depression determines health care-seeking behavior in Chinese patients with dyspepsia and irritable bowel syndrome: a population-based study. Aliment Pharmacol Ther 2002;16:2081-2088.

19. Ahlawat SK, Richard Locke G, Weaver AL, Farmer SA, Yawn BP, Talley NJ. Dyspepsia consulters and patterns of management a population-based study. Aliment Pharmacol Ther 2005;22:251-259. 
20. Howell S, Talley NJ. Does fear of serious disease predict consulting behavior amongst patients with dyspepsia in general practice? Eur J Gastroenterol Hepatol 1999;11:881-886.

21. Drossman DA. The functional gastrointestinal disorders and the Rome III process. Gastroenterology 2006;130:1377-1390.

22. Futagami S, Shimpuku M, Song JM, et al. Nizatidine improves clinical symptoms and gastric emptying in patients with functional dyspepsia accompanied by impaired gastric emptying. Digestion 2012; 86:114-121.

23. McColl K, Murray L, El-Omar E, et al. Symptomatic benefit from eradicating Helicobacter pylori infection in patients with nonulcer dyspepsia. N Engl J Med 1998;339:1869-1874.

24. Portincasa P, Altomare DF, Moschetta A, et al. The effect of acute oral erythromycin on gallbladder motility and on upper gastrointestinal symptoms in gastrectomized patients with and without gallstones: a randomized, placebo-controlled ultrasonographic study. Am J Gastroenterol 2000;95:3444-3451.

25. Svedlund J, Sjödin I, Dotevall G. GSRS--a clinical rating scale for gastrointestinal symptoms in patients with irritable bowel syndrome and peptic ulcer disease. Dig Dis Sci 1988;33:129-134.

26. Doi Y, Minowa M, Okawa M, Uchiyama M. [Development of the Japanese version of the Pittsburgh Sleep Quality Index.] Jpn J Psychiatry Treatment 1998;13:755-763. [Japanese]

27. Spielberger CD, Gorsuch RL, Lushene RE. Manual for State-Trait Anxiety Inventory (Self-Evaluatin Questionnaire). Palo Alto: Consulting Psychologists Press 1970.

28. Fukuhara S, Suzukamo Y. Manual of the SF-8 Japanese version. Institute for Health Outcomes and Process Evaluation research: Kyoto 2004.

29. Agréus L. Socio-economic factors, health care consumption and rating of abdominal symptom severity. A report from the abdominal symptom study. Fam Pract 1993;10:152-163.

30. Westbrook JI, McIntosh J, Talley NJ. Factors associated with consulting medical or non-medical practitioners for dyspepsia: an Australian population-based study. Aliment Pharmacol Ther 2000; 14:1581-1588.

31. Lydeard S, Jones R. Factors affecting the decision to consult with dyspepsia:comparison of consulters and non-consulters. J R Coll Gen Pract 1989;39:495-498.

32. Alander T, Svärdsudd K, Johansson SE, Agréus L. Psychological illness is commonly associated with functional gastrointestinal disorders and is important to consider during patient consultation: a pop- ulation-based study. BMC Med 2005;3:8.

33. Cheng C. Seeking medical consultation: perceptual and behavioral characteristics distinguishing consulters and nonconsulters with functional dyspepsia. Psychosom Med 2000;62:844-852.

34. De La Loge C, Trudeau E, Marquis P, et al. Cross-cultural development and validation of a patient self-administered questionnaire to assess quality of life in upper gastrointestinal disorders: the PAGI-QOL. Qual of Life Res 2004;13:1751-1762.

35. Kaji M, Fujiwara Y, Shiba M, et al. Prevalence of overlaps between GERD, FD and IBS and impact on health-related quality of life. J Gastroenterol Hepatol 2010;25:1151-1156.

36. Miwa $\mathrm{H}$. Life style in persons with functional gastrointestinal disorders - large-scale internet survey of lifestyle in Japan. Neurogastroenterol Motil 2012;24:464-471, e217.

37. Shinozaki M, Fukudo S, Hongo M, et al. High prevalence of irritable bowel syndrome in medical outpatients in Japan. J Clin Gastroenterol 2008;42:1010-1016.

38. Pilichiewicz AN, Horowitz M, Holtmann GJ, Talley NJ, FeinleBisset C. Relationship between symptoms and dietary patterns in patients with functional dyspepsia. Clin Gastroenterol Hepatol 2009;7: 317-322.

39. Pilichiewicz AN, Feltrin KL, Horowitz M, et al. Functional dyspepsia is associated with a greater symptomatic response to fat but not carbohydrate, increased fasting and postprandial CCK, and diminished PYY. Am J Gastroenterol 2008;103:2613-2623.

40. Cuperus P, Keeling PW, Gibney MJ. Eating patterns in functional dyspepsia: a case control study. Eur J Clin Nutr 1996;-50:520-523.

41. Van Oudenhove L, Vandenberghe J, Geeraerts B, et al. Relationship between anxiety and gastric sensorimotor function in functional dyspepsia. Psychosom Med 2007;69:455-463.

42. Lacy BE, Everhart K, Crowell MD. Functional dyspepsia is associated with sleep disorders. Clin Gastroenterol Hepatol 2011;9:410-414.

43. Gonlachanvit S. Are rice and spicy diet good for functional gastrointestinal disorders? J Neurogastroenterol Motil 2010;16:131-138.

44. Geeraerts B, Vandenberghe J, Van Oudenhove L, et al. Influence of experimentally induced anxiety on gastric sensorimotor function in humans. Gastroenterology 2005;129:1437-1444.

45. Kusano M, Zai H, Hosaka H, et al. New frontiers in gut nutrient sensor research: monosodium L-glutamate added to a high-energy, high-protein liquid diet promotes gastric emptying: a possible therapy for patients with functional dyspepsia. J Pharmacol Sci 2010;112:33-36. 
Supplementary Table. Comparison of Each Pittsburgh Sleep Quality Index Score in Tertiary Clinic and Primary Clinic Functional Dyspepsia Outpatients

\begin{tabular}{|c|c|c|c|c|}
\hline & \multicolumn{2}{|c|}{ Teritiary clinic FD outpatient } & \multicolumn{2}{|c|}{ Primary care FD outpatient } \\
\hline & Mean \pm SEM & $P$-value & Mean \pm SEM & $P$-value \\
\hline Subjective sleep quality & $1.14 \pm 0.10$ & 0.425 & $1.32 \pm 0.10$ & 0.543 \\
\hline Sleep latency & $1.00 \pm 0.14$ & 0.001 & $0.74 \pm 0.11$ & 0.031 \\
\hline Sleep duration & $0.65 \pm 0.12$ & 0.054 & $0.76 \pm 0.12$ & 0.178 \\
\hline Habitual sleep efficiency & $0.24 \pm 0.08$ & 0.247 & $0.49 \pm 0.14$ & 0.013 \\
\hline Sleep disturbance & $0.92 \pm 0.09$ & 0.001 & $0.81 \pm 0.08$ & 0.007 \\
\hline Use of sleep medication & $0.71 \pm 0.17$ & 0.001 & $0.80 \pm 0.19$ & 0.001 \\
\hline Daytime dysfunction & $0.69 \pm 0.12$ & 0.660 & $0.66 \pm 0.13$ & 0.567 \\
\hline
\end{tabular}

FD, functional dyspepsia. 